\title{
Seroprevalence of Anti- N-methyl-D-aspartate receptor antibodies in children with seizures of unknown cause
}

\author{
Mohammed Abdulrasol Abdulamer ${ }^{1 \oplus}$, Nebal Waill Saadi ${ }^{1,2}$, \\ Batool Ali Ghalib Yassin ${ }^{3 \oplus}$, Imad Al-Jumaili ${ }^{4 \oplus}$ \\ ${ }^{1}$ Unit of Pediatric Neurology, Children Welfare Teaching Hospital, Medical City Complex, Baghdad, Iraq; Departments of ${ }^{2}$ Pediatrics \\ and ${ }^{3}$ Community, College of Medicine, University of Baghdad, Baghdad, Iraq; ${ }^{4}$ Clinical Chemistry, MSc. Al-Nadaer Clinical Lab. Lab \\ Director, Baghdad, Iraq.
}

\begin{abstract}
Background. Anti- N-methyl-D-aspartate receptor (NMDAR) antibodies were found most probably to be accompanied by seizures, particularly in children and sometimes it may be the sole presenting feature. Therefore, testing these antibodies in children with seizure of unexplained cause might be helpful to identify the spectrum of these antibody-mediated disorders. The objective of this study was to determine the frequency of anti-NMDAR antibodies in patients who presented with seizures of unknown cause.
\end{abstract}

Methods. A case-control study was conducted in two hospitals in Medical City Complex-Baghdad in 2019. Children aged 2-18 years who manifested seizures solely without identified causes were recruited over a period of ten months, with an additional sex- and age-matched control group (forty children in each group). Serum was tested in both groups for anti-NMDAR antibodies.

Results. In the study group, males predominated in ages younger than 5 years. The mean age was 6.6 years and the mean duration since their seizures' onset was 2 months. In contrast to male patients, female patients manifested more focal seizures. Only 5 patients (12.5\%) were positive for Anti- NMDAR antibodies, in contrast to no one in the control group. Significantly, most of the seropositive patients were females $(4,80 \%)$ and showed focal types of seizures $(4,80 \%)$.

Conclusions. This is a preliminary epidemiological study about the prevalence of anti-NMDAR antibodies in a sample of pediatric patients with isolated seizures of unknown cause. Anti-NMDAR antibodies were found to be prevalent in a relatively small proportion of children who presented with seizures of unknown causes. Demographic characteristics of the patients with variable testing status were found to be nearly comparable to the results from other related studies.

Key words: autoimmune, seizures, anti-NMDAR, children.

Epilepsy type of unknown cause is presumed to be due to an underlying cause not diagnosed

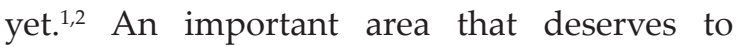
be researched is the presence of specific antibodies associated with seizures. ${ }^{3}$ Those types of epilepsies are either associated with signs and symptoms of encephalitis or

Nebal Waill Saadi

nebalpedneu2013@gmail.com

Received 28th April 2020, revised 13th August 2020,

3rd October 2020, 31st October 2020,

accepted 24th November 2020. presented primarily as recurrent seizures without features of encephalitis. ${ }^{4}$ Symptoms as isolated seizures occur more frequently in children, are commonly of focal type, with an insidious onset or maybe aggressive, very frequent and prolonged with status epilepticus modality. ${ }^{5} \mathrm{~N}$-methyl-D-aspartate receptor (NMDAR) is a neuronal surface antibody and one common type implicated in disorders like epilepsy. ${ }^{6}$ In the human brain, the NMDAR is a ligand of glutamate, the primary excitatory neurotransmitter. Its major role is in synaptic plasticity. Therefore, testing antiNMDAR antibodies in children with seizure 
of unexplained cause might be a significant approach to identify the spectrum of this antibody-associated clinical condition. ${ }^{7}$ It is more sensitive to test for anti-NMDAR antibodies in the cerebrospinal fluid (CSF) than the serum. ${ }^{8}$ Nevertheless, diagnostic sensitivity can be improved by searching for those antibodies in both the serum and CSF. ${ }^{9}$ It is recommended to treat the disease early and extensively because of the intimidating course of the disorder and the recurrent relapses. ${ }^{10}$ The aim of this study was to determine the prevalence of anti-NMDAR antibodies in a group of patients presenting with seizures of unknown cause in comparison to corresponding healthy volunteers.

\section{Material and Methods}

A case - control study was conducted in two hospitals of Medical City Complex (emergency department, outpatient neurology clinic and neurology ward in Children Welfare Teaching Hospital (CWTH), and epilepsy clinic in Baghdad Teaching Hospital) in the period from February to October, 2019. Eighty children were enrolled in the study and divided into two groups: study group and control group with forty children in each. The inclusion criteria were: 1) Age ranged 2 - 18 years; 2) History of seizures, first or recurrent attacks with or without anti-seizure drugs and within the preceding 6 months. The researchers aimed to evaluate the role of anti-NMDAR antibodies in the development of seizure as an isolated feature. Therefore, those children who had additional features like psychiatric or encephalopathic signs and symptoms like behavioural changes, disturbed level of consciousness, or movement disorders which are highly suggestive of autoimmune encephalitis were, particularly excluded. Other exclusion criteria included: 1) evidence of provoked seizures (structural, tumor, infection, metabolic or electrolyte disturbance, or fever $\left.\left(\geq 38{ }^{\circ} \mathrm{C}\right)\right)$, 2) wellestablished electro-clinical epileptic syndrome, 3) personal or family history of autoimmune disorders or epilepsy and 4) patients with preexisting developmental, motor or psychiatric abnormalities. All children and their caregivers were interviewed to explain the objectives of the study and written consent for participation were obtained.

In the study group, a full history and complete physical and neurological examination were performed, and the necessary data were collected using a special form. The gathered data included; gender, age, age of onset of the first attack, semiology of seizures, duration of each attack, type and number of anti-seizure drugs (if present), and if the seizures were controlled or not. Every patient was sent for brain magnetic resonance imaging (MRI) (most had 1.5 Tesla and few had 3 Tesla) and those with either normal study or signal changes not otherwise specified were included in this study. An electroencephalography (EEG) study was also done (standard protocol in all patients except one who had level 2 EEG, and all were assessed by a specialized neurophysiologist), and those with epileptic or normal findings were included.

The classification of the patients' seizures was made by a pediatric neurologist who was blinded to the results of the antibody testing. Classification was made according to the latest ILAE report, so that seizure type at presentation was classified into focal, generalized and unknown onset. This has been made depending on the history taking and also the home video if available. Then the epilepsy type was classified according to the clinical background supported by EEG into focal, generalized, combined focal and generalized and unknown. After that, the patients were tested for antibodies directed against NMDAR with enzyme linked immunoassay (ELISA) by using commercial kits provided by MyBioSource, MBS705691 kits and Mindray MR 96A Elisa Reader used for seroanalysis. Serum samples (3 milliliters) were obtained from each patient, centrifuged, stored at $-20 \mathrm{C}^{\circ}$ to be tested within one month. The cutoff value for this kit was 2.1. Results of values less than 2.1 were considered normal and those equal or more than 2.1 were considered positive. The control group consisted of hospitalized children 
who had serum collected as part of their routine investigations for non-neurological disorders like respiratory, gastrointestinal, hematological and others during the same period of collection of samples from the epileptic patients. They were age- and sex- matched with the study group. Autoimmune disorders were assessed by analysing antinuclear antibodies (ANA). This study was approved by the ethical committee of the Children Welfare Teaching Hospital (IRB: 548, Date 15.12.2018).

Data were analysed using Microsoft excel version 10. Categorical variables were presented as frequency and relative frequency $(\%)$, and continues variables were presented as mean \pm standard deviation. Student T-test was used to test the significant differences between means and chi-square and/or Fischer exact test were used to test the significance of association between categorical variables. P-value of less than 0.05 was considered statistically significant.

\section{Results}

Of the 40 patients enrolled in the study group, $26(65 \%)$ were boys and $14(35 \%)$ were girls. Their age ranged between 2-14 years with a mean age of 6.6 years \pm 3.3 . The mean duration since their seizures' onset was $2 \pm 2.3$ months and the mean age at diagnosis of seizure was $6.4 \pm 3.4$ years. In the study group, the male gender predominated (13 boys versus 2 girls) in the age group younger than 5 years. While both genders presented equally above and equal to 5 years (12 girls versus 13 boys). The association between gender and age of onset of the first seizure in the study group was statistically significant $(\chi 2=4.9, \mathrm{df}=1, \mathrm{P}=0.026)$. Female patients manifested more focal seizures than males (8 (57\%) versus 6 (23\%) respectively), in contrast to male patients who presented with generalized seizures more than females (20 $(77 \%)$ versus $6(43 \%)$ respectively), a result that emphasized statistically significant association between gender and seizure's type ( $\chi 2=4.6$, $\mathrm{df}=1, \mathrm{P}=0.0312$ ). Comparison of anti-NMDAR antibodies results between the study and control groups showed that only five patients $(12.5 \%)$ were positive for anti- NMDAR antibodies, in contrast to no one in the control group (corrected $\mathrm{OR}=12.5$; 95\% CI (0.6-216.7)). The majority of patients with positive anti-NMDAR antibodies were females (4 (80\%) versus $1(20 \%))$, in contrast to male predominance in the patients with negative anti-NMDAR antibodies (25 $(71.3 \%)$ versus $10(28.7 \%))$, with a statistically significant association $(\chi 2=5.08, \mathrm{df}=1, \mathrm{P}=0.024)$.

Table I. Variable characteristics of the study group stratified by anti-NMDAR antibody status.

\begin{tabular}{|c|c|c|c|c|}
\hline \multicolumn{2}{|l|}{ Variable } & $\begin{array}{c}\text { Anti-NMDA +ve } \\
\text { No. }(\%)\end{array}$ & $\begin{array}{c}\text { Anti-NMDA -ve } \\
\text { No. }(\%)\end{array}$ & $\mathrm{p}$ value \\
\hline \multicolumn{5}{|l|}{ Age (years) } \\
\hline Range & & $3-12$ & $2-14$ & \multirow{2}{*}{0.76} \\
\hline Mean \pm SD & & $7.1 \pm 3.7$ & $6.6 \pm 3.3$ & \\
\hline \multicolumn{5}{|c|}{ Age at diagnosis (years) } \\
\hline Range & & $3-12$ & $1.9-13.9$ & \multirow{2}{*}{0.7} \\
\hline Mean \pm SD & & $7.03 \pm 3.8$ & $6.4 \pm 3.4$ & \\
\hline \multicolumn{5}{|c|}{ Duration since seizure onset (months) } \\
\hline Range & & $0.25-2$ & $0.25-10$ & \multirow{2}{*}{0.3} \\
\hline Mean \pm SD & & $0.95 \pm 0.7$ & $2.2 \pm 2.5$ & \\
\hline \multirow{2}{*}{ Type of seizure } & Focal & $4(80)$ & $10(29)$ & \multirow{2}{*}{0.024} \\
\hline & Generalized & $1(20)$ & $25(71)$ & \\
\hline \multirow{2}{*}{$\begin{array}{l}\text { Treatment with anti- } \\
\text { seizure drugs }\end{array}$} & Not treated & $1(20)$ & $15(43)$ & \multirow{2}{*}{0.6} \\
\hline & Treated & $4(80)$ & $20(57)$ & \\
\hline
\end{tabular}


The distribution of the study group by age, age at onset of first seizure and duration since seizure onset according to the results of anti-NMDAR antibodies is shown in Table I. The mean of both patients' ages and age of first seizure were higher in those with antibody positive result, yet, it was not statistically significant $(P$ value 0.76 and 0.7 respectively). Anti-NMDAR positive patients presented earlier (within one month) with no significant statistical result ( $P$ value 0.3 ). The percentage of focal seizures was significantly higher in patients with antibody positive results $(80 \%)$ compared to those with negative results. This was statistically significant (P-value $=0.024)$ as shown in Table I. Before testing the anti-NMDAR antibodies, the usual treatment of children in the study group were anti-seizure drugs. No immunosuppressive or immune modulators were administered before or after obtaining the results in anti-NMDAR positive patients. The association between antiNMDAR status and the use of anti-seizure drugs is described in Table I, and was found to be non-significant $(P=0.6)$, yet a higher percentage of those with positive anti-NMDAR status were treated.

All patients in the study group had normal EEG and brain MRI results. Demographic and clinical characteristics of the five patients with positive anti-NMDAR status is presented in Table II. Only one patient presented at a relatively young age (3 years) with only one attack of generalized tonic-clonic seizure. His EEG was reported as normal and he was on no anti-seizure medications. The other four patients were 5 years and older, of female gender, had focal type of epilepsy with frequent focal seizures and fairly partial response to anti-seizure medications. On one-month follow up, three patients became seizure free, while contact was lost with the other two patients. Anti-NMDAR antibodies were not tested again.

\section{Discussion}

In the human brain, NMDAR is a ligand of glutamate, the primary excitatory neurotransmitter. Its major function is in synaptic plasticity, substantial for memory function and excitotoxicity that is implicated in a number of diseases like epilepsy and Alzheimer's. It is detected all around the central nervous system (CNS), in approximately $80 \%$ of cortical neurons. Several subtypes of NMDAR are found, each one is made up of two N1 subunits and either two N2 or two N3

Table II. Demographic and clinical characteristics of patients with positive anti-NMDAR antibodies.

\begin{tabular}{|c|c|c|c|c|c|c|c|c|}
\hline Patient & $\begin{array}{l}\text { Age } \\
\text { (years) }\end{array}$ & Gender & $\begin{array}{l}\text { Time of } \\
\text { sampling } \\
\text { since onset } \\
\text { of seizure }\end{array}$ & Type of seizure & $\begin{array}{l}\text { Type of } \\
\text { epilepsy }\end{array}$ & $\begin{array}{l}\text { MRI \& } \\
\text { EEG }\end{array}$ & ASD & Follow up \\
\hline 1 & 5.5 & Female & 2 months & $\begin{array}{l}\text { Focal to bilateral } \\
\text { tonic-clonic }\end{array}$ & Focal & Normal & $\begin{array}{l}\text { Levetiracetam } \\
20 \mathrm{mg} / \mathrm{kg} / \text { day }\end{array}$ & $\begin{array}{l}\text { Seizure free / } \\
\text { On treatment }\end{array}$ \\
\hline 2 & 12 & Female & 1 month & Focal & Focal & Normal & $\begin{array}{l}\text { Oxcarbazepine } \\
15 \mathrm{mg} / \mathrm{kg} / \text { day }\end{array}$ & Lost contact \\
\hline 3 & 10 & Female & 2 weeks & Focal tonic & Focal & Normal & $\begin{array}{l}\text { Carbamazepine } \\
10 \mathrm{mg} / \mathrm{kg} / \text { day }\end{array}$ & Lost contact \# \\
\hline 4 & 3 & Male & 2 days & $\begin{array}{l}\text { Attack of } \\
\text { Generalized tonic } \\
\text { seizure }\end{array}$ & $\begin{array}{l}\text { Non } \\
\text { applicable }\end{array}$ & Normal & None & $\begin{array}{l}\text { Seizure free / } \\
\text { No treatment }\end{array}$ \\
\hline 5 & 5 & Female & 1 month & Focal & Focal & Normal & $\begin{array}{l}\text { Levetiracetam } \\
20 \mathrm{mg} / \mathrm{kg} / \text { day }\end{array}$ & $\begin{array}{l}\text { Seizure free / } \\
\text { No treatment }\end{array}$ \\
\hline
\end{tabular}

MRI: magnetic resonance imaging, EEG: electroencephalography, ASD: anti-seizure drugs

\# Last contact was before 4 months during that time she was free of seizure and discontinued her treatment 
subunits. ${ }^{11}$ The construction of the receptor is formed generally of an extracellular part for ligand binding and an ion channel which allows the entrance of cations in and out of the cells controlled by the force of the individual electrochemical gradients. Once the ligand is tied up to its sites on the receptor, modifications in configurations in the receptor protein allows the entry of $\mathrm{Na}$ and $\mathrm{Ca}$ in and the emergence of $\mathrm{K}$ out of the cell which causes depolarization and neuronal excitation. ${ }^{12}$ The activity status of the NMDAR may determine the clinical profile in the way that over-activity is causing excitotoxicity with subsequent features like epilepsy, stroke, and dementia, while underactivity results in symptoms of schizophrenia. ${ }^{13}$ Immunoglobulin $G$ antibodies are found to cause epilepsy when it is directed toward GluN1 subunit of the NMDAR resulting in extended openings of that receptor and causing acquisition-of-function. ${ }^{14}$

The occurrence of antibodies against NMDAR in patients with encephalitis has been the focus of several studies, yet very little has been reported about their prevalence in seizures of unknown cause in children. A study taken place in the UK in 2010 reported 4 (9\%) samples collected from patients (44) tested positive for anti-NMDAR antibodies, two belonged to males (both aged 23) with a 4-year history of drug-resistant temporal lobe epilepsy, and two females (aged 17 and 33 years) who presented with an acuteonset of complex partial status epilepticus, had had minimal or no cognitive involvement and did not develop any movement disorders or other features consistent with the later stages of the disease.${ }^{15}$ Positive result of anti-NMDAR antibodies were also reported in studies conducted in Sydney by Suleiman et al. ${ }^{6}(6 \%$ of pediatric patients with seizures of unknown cause), ${ }^{6}$ and in the UK in 2013 (1.7\% of patients older than 16 years with established and newly diagnosed epilepsy). ${ }^{16}$ In the current study, the prevalence of anti-NMDA antibodies in patients with seizures of unknown causes was found to be $12.5 \%$, which showed significant statistical feature $(\mathrm{OR}=12.5)$, yet it cannot be applied to the general population as the CI was $0.6-216.7$, which may be related to the small size of the sample and we might have obtained different percentage if both CSF and serum were tested for anti-NMDAR antibodies as it is known that serum testing has lower sensitivity than CSF. ${ }^{17}$ This heterogeneity of anti-NMDAR antibodies positivity among studies might be due to different lab techniques used to assess the autoantibodies and different sampling time.

The current study showed that the male gender predominated in the study group, at ages below and above 5 years. Nevertheless, the number of female patients increased gradually and approached that of males after 5 years of age (male: female ratio was 6.5:1 versus 1.1:1 respectively). The latter may be attributed to the hormonal changes that may play a significant role in female epilepsy near adolescence. The same was reported by studies conducted in Western Nepal in 2013 by Adhikari et al. ${ }^{18}$ and in China in 2016 by Mwipopo et al., ${ }^{19}$ where prospective and retrospective analyses were taken place for children admitted with acute seizures. Sex differences in epilepsy and seizure are not always clear. Particularly if considering all epilepsy types. Documented effects of sex on seizures have been reported, and numerous effects of gonadal steroids have been shown throughout the rodent brain. There is a broad agreement among studies that males have greater exposure to risk factors for lesional epilepsy and acute symptomatic seizures. $^{20,21}$ However, inconsistencies were reported regarding epilepsy prevalence in different genders. Some cohorts showed a higher prevalence of epilepsy in men while others showed an opposite result. This may be due to pooling subjects with different epilepsy syndromes or neuropathology. ${ }^{22}$ The small sized sample of the study group, causes difficulty to explain males predominance in regard to seizure prevalence. Gender difference in children with anti NMDAR encephalitis were reported to be in favour of the female sex..$^{23-25}$

The mean age of first seizure presentation was 6.4 $\pm 3.4 \mathrm{SD}$ years and this was relatively 
higher than most of the studies that discuss the epidemiology of epilepsy, ${ }^{18,19,26}$ which may be explained by the fact that the current study has included a wider range of ages (2-18 years) and also excluded patients with febrile seizures, CNS infection, congenital malformation, metabolic and electro-clinical syndromes which constitute the major causes of epilepsy in younger children.

Generalized seizures predominated in our cohort (63\%), which agreed with that reported in Mwipopo et al., ${ }^{19}$ Chen et al. ${ }^{26}$ and the metaanalysis in 2017 by Fiest et al. ${ }^{27}$, but disagreed with other studies like Berg et al..$^{28}$ and Camfield et al. ${ }^{29}$ Hauser has suggested in Pellock's Pediatric Epilepsy that in children both single, unprovoked seizures and acute symptomatic seizures are predominantly generalized. This difference in distribution by seizure type may be related to the inclusion criteria used for each study. ${ }^{30}$

Most of our female patients presented with focal seizures compared to the males (P-value $=0.0312)$. However, the few numbers of patients in the study group suggested that the results need to be interpreted with caution. Pituitary and gonadal hormones are known to affect cortical excitability. Intravenous infusion of estrogen directly applied in to the cerebral cortex can activate seizures and interictal discharges. This is partially caused by alteration of cell membrane permeability to calcium, reduction of chloride influx through the gamma-aminobutyric acid (GABA)-A receptor, and glutamate agonist action of estrogen in the hippocampus. ${ }^{31}$ Variable results were reported about sex difference for seizure types, some showed that idiopathic generalized epilepsy or multiple seizure types are more common in females. ${ }^{32}$ Other reported that atonic seizures were more common in males with generalized epilepsy, and that autonomic, visual, and psychic symptoms associated with nonacquired focal epilepsy, were more common in females. ${ }^{33}$ The current study reported no one in the control group with positive antiNMDAR antibodies, in contrast to other studies which showed positivity in the serum of healthy persons as in USA in $2014(0.4 \%)$ and in Germany in $2013(3 \%))^{8,34}$

Most of the patients with positive results were females $(80 \%)$ with a female to male ratio of $4: 1$, and showed statistical significance. This was agreed by a study conducted in Spain in 2013 by Armangue et al. ${ }^{16}$ and another one in the UK in 2010 by Vincent et al. ${ }^{35}$ It may be related to the fact that the risk of autoimmune diseases increases in females. Male predominance was reported in Brenner et al. ${ }^{16}$ in 2013 (male: female ratio 1.3:1) while both Suleiman et al. ${ }^{6}$ and Irani et al. ${ }^{15}$ found a 1:1 ratio. Female predominance was also reported in studies surveying children with anti-NMDAR encephalitis. ${ }^{23-25}$

The mean duration since the first seizure onset and timing of the samples was 28.5 days for the positive patients versus 66 days for the negative patients with no statistically significant difference. A similar result was found by Suleiman et al. ${ }^{6}$ (17.5 days for positive tests versus 40 days for negative results with no statistical differences). Vincent et al. ${ }^{35}$ has proposed that these antibodies usually present at their nadir early in the disease course and may gradually decrease thereafter even without treatment. ${ }^{35}$ Based on this, those patients with negative results who presented later may have had positive anti-NMDA antibodies if they had been tested earlier.

The mean age at first seizure presentation in the positive patients was 7.1 years versus 6.4 years for those with negative results. This was higher than that in the study of Suleiman et al. ${ }^{6}$ (4.4 years) which might be attributed to the larger age range collected in that study, and it was much lower than that found in the samples collected by Irani et al. ${ }^{15}$ (23 years in two samples, 17 years and 33 years in another two samples) which could be explained by referring the samples of the patients from adult neurologists. Generally, studies reported older ages of patients presenting with antiNMDAR antibodies encephalitis as in studies by Armangue et al., ${ }^{37}$ Zekeridou et al., ${ }^{5}$ Remy et 
al. ${ }^{23}$ and Florance et al. ${ }^{36}$,

Eighty percent of patients with positive results had focal seizures. This may be related to the inflammatory nature of this disorder affecting certain areas in the brain more than others. This was similar to the results found by Brenner et al. ${ }^{16}$ and Suleiman et al. ${ }^{6}$ Other studies discussing anti-NMDA associated autoimmune encephalitis also reported the predominance of focal seizures in the patients. ${ }^{5,36,37}$

No difference was found between seropositive and seronegative groups regarding the use of anti-seizure drugs as shown in Table I. These medications have little or no effects on these antibody - associated disorders. ${ }^{38}$ Additionally, most of our positive patients were newly diagnosed and the effects of these medications can't be evaluated appropriately. Epilepsy itself and antiepileptic drugs are reported to alter immune responses, and it is not clear which autoantibodies arise as a consequence and which are causative. ${ }^{39}$

This is a preliminary epidemiological study about the prevalence of anti-NMDAR antibodies in a sample of pediatric patients with isolated seizures of unknown cause. Anti-NMDAR antibodies were prevalent in a small proportion of children with isolated seizures, who lacked other familiar manifestations of autoimmune encephalitis. The demographic characteristics of children with positive anti-NMDAR antibodies was nearly similar to other studies. We could neither duplicate the testing in those who showed positive results, because of financial restraints, nor be able to treat them, as we were not sure of the cause - effect relationship, and the manifestations (seizures) of the antiNMDAR positive patients were not persistent, progressive or reluctant to conventional antiseizure medications, to encourage the start of immune suppressive medications. The small sample size, the shorter follow up duration and the case- control statistical design of this study are the main limitations, so we were unable to reach a strong conclusion, in regard of measuring a cause- effect relationship. The latter necessitates a cohort study that includes longer follow up. Instead, the prevalence of the anti - NMDAR antibodies were investigated in this group of patients.

Furthermore, there is a need for larger prospective analysis of paired serum and CSF anti-NMDAR antibody titer in children with isolated seizures of unknown cause to optimize the laboratory diagnostic sensitivity and characterize the true prevalence of these antibodies among those patients. In conclusion, it is recommended to consider and screen for autoimmune etiologies of epilepsy, particularly in epidemiologically typical circumstances.

\section{Acknowledgements}

We are grateful to the patients and their families for their informed consent and agreement to participate in this study.

\section{Author contribution}

The authors confirm contribution to the paper as follows: study conception and design: Nebal Waill Saadi, Mohammed Abdulrasol Abdulamer; data collection: Mohammed Abdulrasol Abdulamer; analysis and interpretation of results: Imad Al-Jumaili, Batool Ali Ghalib Yassin, Nebal Waill Saadi, Mohammed Abdulrasol Abdulamer; draft manuscript preparation: Nebal Waill Saadi, Mohammed Abdulrasol Abdulamer, Batool Ali Ghalib Yassin. All authors reviewed the results and approved the final version of the manuscript.

\section{Ethical approval}

This study was approved by the ethical committee of the Children Welfare Teaching Hospital (IRB: 548, Date 15.12.2018).

\section{Source of funding}

None. 


\section{Conflict of interest}

The authors declare no conflict of interest.

\section{REFERENCES}

1. Panayiotopoulos CP. The new ILAE report on terminology and concepts for the organization of epilepsies: critical review and contribution. Epilepsia 2012; 53: 399-404.

2. van Campen JS, Jansen FE, Brouwer OF, Nicolai J, Braun KP. Interobserver agreement of the old and the newly proposed ILAE epilepsy classification in children. Epilepsia 2013; 54: 726-732.

3. Suleiman J, Dale RC. The recognition and treatment of autoimmune epilepsy in children. Dev Med Child Neurol 2015; 57: 431-440.

4. Dubey D, Alqallaf A, Hays R, et al. Neurological autoantibody prevalence in epilepsy of unknown etiology. JAMA Neurol 2017; 74: 397-402.

5. Zekeridou A, Karantoni E, Viaccoz A, et al. Treatment and outcome of children and adolescents with N-methyl-D-aspartate receptor encephalitis. J Neurol 2015; 262: 1859-1866.

6. Suleiman J, Wright S, Gill D, et al. Autoantibodies to neuronal antigens in children with new-onset seizures classified according to the revised ILAE organization of seizures and epilepsies. Epilepsia 2013; 54: 2091-2100.

7. Suleiman J, Brilot F, Lang B, Vincent A, Dale RC. Autoimmune epilepsy in children: case series and proposed guidelines for identification. Epilepsia 2013; 54: 1036-1045.

8. Gresa-Arribas N, Titulaer MJ, Torrents A, et al. Antibody titres at diagnosis and during follow-up of anti-NMDA receptor encephalitis: a retrospective study. Lancet Neurol 2014; 13: 167-177.

9. Suh-Lailam BB, Haven TR, Copple SS, Knapp D, Jaskowski TD, Tebo AE. Anti-NMDA-receptor antibody encephalitis: performance evaluation and laboratory experience with the anti-NMDA-receptor IgG assay. Clin Chim Acta 2013; 421: 1-6.

10. Irani SR, Bien CG, Lang B. Autoimmune epilepsies. Curr Opin Neurol 2011; 24: 146-153.

11. Jewett BE, Thapa B. Physiology, NMDA Receptor. PMID: 30137779 Bookshelf ID: NBK519495. In: StatPearls [Internet] Treasure Island (FL): StatPearls Publishing, 2021. Available at: https://pubmed.ncbi. nlm.nih.gov/30137779/ (Accessed on April 2, 2021).

12. Rogawski MA. The NMDA receptor, NMDA antagonists and epilepsy therapy. A status report. Drugs 1992; 44: 279-292.
13. Lau CG, Zukin RS. NMDA receptor trafficking in synaptic plasticity and neuropsychiatric disorders. Nat Rev Neurosci 2007; 8: 413-426.

14. Seebohm G, Piccini I, Strutz-Seebohm N. Paving the way to understand autoantibody-mediated epilepsy on the molecular level. Front Neurol 2015; 6: 149.

15. Irani SR, Bera K, Waters $\mathrm{P}$, et al. N-methylD-aspartate antibody encephalitis: temporal progression of clinical and paraclinical observations in a predominantly non-paraneoplastic disorder of both sexes. Brain 2010; 133(Pt 6): 1655-1667.

16. Brenner T, Sills GJ, Hart Y, et al. Prevalence of neurologic autoantibodies in cohorts of patients with new and established epilepsy. Epilepsia 2013; 54: 1028-1035.

17. Graus F, Titulaer MJ, Balu R, et al. A clinical approach to diagnosis of autoimmune encephalitis. Lancet Neurol 2016; 15: 391-404.

18. Adhikari S, Sathian B, Koirala DP, Rao KS. Profile of children admitted with seizures in a tertiary care hospital of Western Nepal. BMC Pediatr 2013; 13: 43.

19. Mwipopo EE, Akhatar S, Fan P, Zhao D. Profile and clinical characterization of seizures in hospitalized children. Pan Afr Med J 2016; 24: 313.

20. McHugh JC, Delanty N. Epidemiology and classification of epilepsy: gender comparisons. Int Rev Neurobiol 2008; 83: 11-26.

21. Christensen J, Kjeldsen MJ, Andersen H, Friis ML, Sidenius P. Gender differences in epilepsy. Epilepsia 2005; 46: 956-960.

22. Kim DW, Lee SY, Chung SE, Cheong HK, Jung KY, Korean Epilepsy Society. Clinical characteristics of patients with treated epilepsy in Korea: a nationwide epidemiologic study. Epilepsia 2014; 55: 67-75.

23. Remy KE, Custer JW, Cappell J, et al. Pediatric Anti-N-methyl-D-aspartate receptor encephalitis: a review with pooled analysis and critical care emphasis. Front Pediatr 2017; 5: 250.

24. Sai Y, Zhang X, Feng M, Tang J, Liao H, Tan L. Clinical diagnosis and treatment of pediatric anti $\mathrm{N}$ methyl D aspartate receptor encephalitis: a single center retrospective study. Exp Ther Med 2018; 16: 1442-1448.

25. Zhang M, Li W, Zhou S, et al. Clinical features, treatment, and outcomes among Chinese children with anti-methyl-D-aspartate receptor (antiNMDAR) encephalitis. Front Neurol 2019; 10: 596. 
26. Chen CY, Chang YJ, Wu HP. New-onset seizures in pediatric emergency. Pediatr Neonatol 2010; 51: 103111.

27. Fiest KM, Sauro KM, Wiebe S, et al. Prevalence and incidence of epilepsy: a systematic review and metaanalysis of international studies. Neurology 2017; 88: 296-303.

28. Berg AT, Shinnar S, Levy SR, Testa FM. Newly diagnosed epilepsy in children: presentation at diagnosis. Epilepsia 1999; 40: 445-452.

29. Camfield P, Camfield C. Incidence, prevalence and aetiology of seizures and epilepsy in children. Epileptic Disord 2015; 17: 117-123.

30. Hauser WA. Epidemiology of epilepsy in children. In: Pellock JM, Nordli DR Jr, Sankar R, Wheless JW, (eds). Pellock's Pediatric Epilepsy. (4th ed). New York, NY: Demos Medical Publishing, 2017: 177-205.

31. Boggs JG. Women's Health and Epilepsy. Sex Hormones and Epilepsy. Medscape. Updated December 2018. Available at: https://emedicine. medscape.com/article/1186482-overview\#a2. (Accessed on April 2, 2020).

32. Kishk N, Mourad H, Ibrahim S, Shamloul R, AlAzazi A, Shalaby N. Sex differences among epileptic patients: a comparison of epilepsy and its impacts on demographic features, clinical characteristics, and management patterns in a tertiary care hospital in Egypt. Egypt J Neurol Psychiatr Neurosurg 2019; 55: 39.
33. Carlson C, Dugan P, Kirsch HE, Friedman D, The EPGP Investigators. Sex differences in seizure types and symptoms. Epilepsy Behav 2014; 41: 103-108.

34. Viaccoz A, Desestret V, Ducray F, et al. Clinical specificities of adult male patients with NMDA receptor antibodies encephalitis. Neurology2014; 82: 556-563.

35. Vincent A, Irani SR, Lang B. The growing recognition of immunotherapy-responsive seizure disorders with autoantibodies to specific neuronal proteins. Curr Opin Neurol 2010; 23: 144-150.

36. Florance NR, Davis RL, Lam C, et al. Anti-N-methylD-aspartate receptor (NMDAR) encephalitis in children and adolescents. Ann Neurol 2009; 66: 1118.

37. Armangue T, Titulaer MJ, Málaga I, et al; Spanish Anti-N-methyl-D-Aspartate Receptor (NMDAR) Encephalitis Work Group. Pediatric anti-N-methylD-aspartate receptor encephalitis-clinical analysis and novel findings in a series of 20 patients. J Pediatr 2013; 162: 850-856.e2.

38. Cabezudo-García P, Mena-Vázquez N, VillagránGarcía M, Serrano-Castro PJ. Efficacy of antiepileptic drugs in autoimmune epilepsy: a systematic review. Seizure 2018; 59: 72-76.

39. Palace J, Lange B. Epilepsy: an autoimmune disease? J Neurol Neurosurg Psychiatry 2000; 69: 711-714. 